The difference may be partly accounted for by the factors that influence infectivity of the two viruses. Hepatitis B is much more likely to be transmitted from mother to infant if there is a high concentration of the virus in the mother's blood. This explains the ethnic differences that are observed-for example, the transmission rate is over $70 \%$ in Chinese women but less than $10 \%$ in white women. This ethnic difference does not seem to apply to hepatitis $\mathrm{C}$ infection.

Alcohol intake and obesity are both thought to be associated with more severe hepatitis $\mathrm{C}$, although the exact interaction is unknown. Advanced liver disease, for example, is far worse in people infected with hepatitis $\mathrm{C}$ who also have a high alcohol intake than in those with a low intake. About half of patients with hepatitis B infections respond to interferon compared with $15 \%$ with hepatitis C. Ongoing trials of interferon and antivirals together may prove more fruitful. Although infection with hepatitis $\mathrm{C}$ virus does not necessarily cause abnormal liver function, precirrhotic damage confirmed by biopsy is one reason for starting treatment with interferon.

Abi Berger Science editor, BMJ

\title{
Deliberate self harm: systematic review of efficacy of psychosocial and pharmacological treatments in preventing repetition
}

Keith Hawton, Ella Arensman, Ellen Townsend, Sandy Bremner, Eleanor Feldman, Robert Goldney, David Gunnell, Philip Hazell, Kees van Heeringen, Allan House, David Owens, Isaac Sakinofsky, Lil Träskman-Bendz

\begin{abstract}
Objective: To identify and synthesise the findings from all randomised controlled trials that have examined the effectiveness of treatments of patients who have deliberately harmed themselves.

Design: Systematic review of randomised controlled trials of psychosocial and physical treatments. Studies categorised according to type of treatment. When there was more than one investigation in a particular category a summary odds ratio was estimated with the Mantel-Haenszel method.

Setting: Randomised trials available in electronic databases in 1996, in the Cochrane Controlled Trials Register in 1997, and from hand searching of journals to 1997.

Subjects: Patients who had deliberately harmed themselves shortly before entry into the trials with information on repetition of behaviour. The included trials comprised 2452 randomised participants with outcome data.

Main outcome measure: Repetition of self harm.

Results: 20 trials reported repetition of self harm as an outcome variable, classified into 10 categories.

Summary odds ratio (all for comparison with standard aftercare) indicated reduced repetition for problem solving therapy $(0.73 ; 95 \%$ confidence interval 0.45 to 1.18) and for provision of an emergency contact card in addition to standard care $(0.45 ; 0.19$ to 1.07$)$. The summary odds ratios were 0.83 (0.61 to 1.14 ) for trials of intensive aftercare plus outreach and 1.19 (0.53 to 2.67) for antidepressant treatment compared with placebo. Significantly reduced rates of further self harm were observed for depot flupenthixol versus placebo in multiple repeaters $(0.09 ; 0.02$ to 0.50$)$ and for dialectical behaviour therapy versus standard aftercare $(0.24 ; 0.06$ to 0.93 ).

Conclusion: There remains considerable uncertainty about which forms of psychosocial and physical treatments of patients who harm themselves are most effective. Further larger trials of treatments are needed.
\end{abstract}

\section{Introduction}

Prevention of suicide is now included in health policy initiatives in several countries, and reduction in suicidal behaviour, both fatal and non-fatal, is part of the Health for All targets of the World Health Organisation. ${ }^{1}$ In the United Kingdom, reduction in the number of suicides is a central theme in the government's Health of the Nation strategy for England. ${ }^{2}$ There is, however, a considerable lack of information as to which preventive strategies are effective. ${ }^{3}$ Improvement of outcome after deliberate self harm is an important focus because at least $1 \%$ of patients presenting to general hospitals in the United Kingdom after deliberate self harm kill themselves within a year and 3-5\% do so within 5-10 years. A history of multiple episodes of deliberate self harm is a particular risk factor. ${ }^{4}$ Higher rates of suicide after deliberate self harm have been reported from other countries. $^{56}$ About half of all people who kill themselves have a history of deliberate self harm, an episode having occurred within the year before death in $20-25 \% .^{78}$

It would be difficult to investigate the effectiveness of intervention strategies after deliberate self harm in terms of subsequent actual suicides because extremely large populations of patients would be required. Repetition of deliberate self harm is, however, a reasonable proxy measure because of its strong associations with suicide. It is also in itself an important outcome because it occurs frequently, ${ }^{90}$ indicates persistent distress, and results in considerable healthcare costs. Deliberate self harm is common in Europe ${ }^{11}$ and in other parts of the world, ${ }^{12}{ }^{13}$ especially in young people. Recent marked increases in rates of deliberate self harm in the United Kingdom, ${ }^{14}{ }^{15}$ with a currently estimated 140000 hospital referrals in England and Wales, ${ }^{10}$ have highlighted the need for effective aftercare strategies.

Descriptive reviews of treatment outcomes in patients who deliberately harm themselves have been

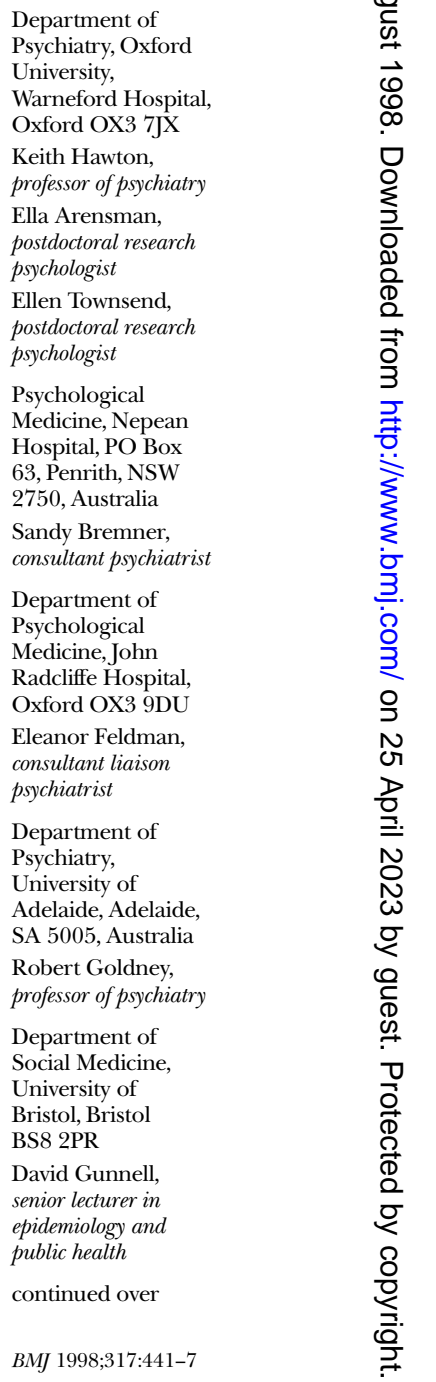


Discipline of

Psychiatry, Faculty

of Medicine and

Health Sciences,

University of

Newcastle,

Callaghan, NSW

2308, Australia

Philip Hazell,

professor of psychiatry

Department of

Psychiatry,

University Hospital,

9000 Ghent,

Belgium

Kees van

Heeringen,

professor of psychiatry

Division of

Psychiatry and

Behavioural

Sciences, School of

Medicine,

University of Leeds,

Leeds LS2 9LT

Allan House,

senior lecturer in

psychiatry

David Owens,

senior lecturer in

psychiatry

Clarke Institute of

Psychiatry, Toronto,

Canada MST IR8

Isaac Sakinofsky,

professor of psychiatry

Department of

Psychiatry,

University Hospital,

22185 Lund,

Sweden

Lil Träskman-

Bendz,

professor of psychiatry

Correspondence to:

Professor Hawton

Keith.Hawton@

psychiatry.ox.ac.uk published previously but have not included systematic screening of the literature, quality ratings, and meta-analysis, ${ }^{16-18}$ and have been based on heterogeneous groupings of treatments which do not inform clinical practice. $^{19}$

We conducted a systematic review of the worldwide literature regarding treatment studies of patients who deliberately harm themselves. We identified all randomised controlled trials evaluating psychosocial or physical treatments and conducted a meta-analysis to compare the effects of specific treatments on repetition of deliberate self harm with those of control or comparison treatments to identify the most effective interventions.

\section{Methods}

Identification of relevant trials-We carried out a literature search using the following electronic databases: Medline 1966 to May 1996; PsycLit 1974 to August 1996; Embase 1980 to November 1996; and the Cochrane Controlled Trials Register (No 4, 1997). ${ }^{20}$ A wide range of keywords to indicate attempted suicide and a standard search strategy, developed for the Medline database by the Cochrane Collaboration, was used to identify relevant randomised controlled trials. A shorter version of this search strategy was used to perform searches on PsycLit and Embase (details of search strategies available from the authors). In addition, we hand searched 10 journals in the specialty of psychiatry and psychology that had not been searched within the Cochrane Collaboration, including all the English language journals concerned with suicide.

Inclusion criteria-We included studies in the review if they met the following criteria: study participants had to have engaged in deliberate self harm (self poisoning or self injury) shortly before entry into the trial; trials must have reported repetition of deliberate self harm as an outcome measure; and study participants had to have been randomised to treatment and control groups. As long as these three criteria were met we included papers reporting any comparison between different types of treatment, including comparisons with standard (that is, routine) aftercare. When details of standard aftercare were not provided, we attempted to obtain these from the authors.

Grouping of studies-Studies that shared similar treatment strategies were grouped by consensus of the reviewers, blind to the outcome data. The first category (problem solving therapy $v$ standard aftercare) included studies in which participants in the experimental group were offered some form of problem solving therapy which was compared with standard aftercare. Standard aftercare, both here and in other categories, included the usual range of treatment options that were available in routine care at the time in each setting. The second group (intensive intervention plus outreach $v$ standard aftercare) included studies in which the patients in the experimental group had greater access to therapists than in standard care and where efforts were made to keep contact with patients through some form of outreach (for example, home based treatment either as standard or for those patients who defaulted on appointments at a clinic). The third group (emergency card $v$ standard aftercare) included studies in which patients in the experimental group, in addition to being offered standard aftercare, were given an emergency contact card with which they had 24 hour access to emergency advice from a psychiatrist $^{21}$ or could admit themselves to hospital. ${ }^{22}$ In only one other group (antidepressant medication $v$ placebo) was there more than one trial. The remainder of the studies are reported singly.

Data extraction and quality assessment-Data were extracted independently by two reviewers. The quality of the papers was rated by two independent reviewers blind to authorship, according to the recommended Cochrane criteria for quality assessment. ${ }^{23}$ This rating system is influenced by the finding that the quality of concealment of random allocation can affect the results of trials. ${ }^{24}$ Studies were assigned a quality score from 1 (poorest quality) to 3 (best quality). Thus, trials rated as inadequately concealed (for example, via alternation or reference to an open random number table) were given a score of 1 . Trials that did not give adequate details about how the randomisation procedure was carried out were given a score of 2 . Trials that were deemed to have taken adequate measures to conceal allocation (for example, serially numbered, opaque, sealed envelopes; numbered or coded bottles or containers) were given a score of 3 . We contacted authors of trials for more information when the concealment of allocation was not clearly reported (that is, when trials were initially in category 2). Blinding of observers was rated according to whether it was absent or unclear, reported but without details, and fully reported.

Statistical methods-Summary odds ratios were calculated with RevMan 3.0 software ${ }^{25}$ with the MantelHaenszel method. Heterogeneity was tested with a $\chi^{2}$ test.

\section{Results}

Twenty studies were identified through the combined search strategies as eligible for inclusion in the study. All reports had been published, although two studies had not been published in full. ${ }^{16}{ }^{26}$ The full report of one trial was provided by the author in the form of an unpublished manuscript ${ }^{16}$ and a detailed report of the other was obtained from conference proceedings. ${ }^{26}$ Four studies had been reported in more than one publication. ${ }^{16}{ }^{26-28}$ One further randomised controlled trial of patients who deliberately harmed themselves was identified, but this did not include repetition of deliberate self harm as an outcome variable. ${ }^{29}$ We were unable to obtain this information from the authors of this trial.

The trials identified were grouped as described in the methods section. Table 1 summarises the 20 trials included in the review, their groupings, details of participants (sex and the proportion with a history of self harm-"repeaters"), the interventions used, and the quality of concealment scores. Only one trial was specifically of adolescents. ${ }^{22}$

The assessment of quality of concealment of allocation (table 1) resulted in 13 trials being given a score of 3 (for adequate concealment), three trials being given a score of 2 (unclear concealment), and four trials being given a score of 1 (inadequate concealment). Blinding of assessors was not stated or 
Table 1 Summary of participants, interventions, follow up period, size of trial, and quality of concealment of allocation

\begin{tabular}{|c|c|c|c|c|c|}
\hline Study & Details of participants & Interventions & $\begin{array}{l}\text { No randomised } \\
\text { (No lost to } \\
\text { follow up or } \\
\text { excluded) }\end{array}$ & $\begin{array}{l}\text { Follow up } \\
\text { period }\end{array}$ & $\begin{array}{l}\text { Quality of } \\
\text { concealment } \\
\text { of allocation* }\end{array}$ \\
\hline \multicolumn{6}{|c|}{ Problem solving therapy $v$ standard aftercare } \\
\hline $\begin{array}{l}\text { Gibbons et al } \\
\quad(\mathrm{UK}, 1978)^{30}\end{array}$ & $\begin{array}{l}\text { Patients over } 17 \text { years who presented to A\&E } \\
\text { department after deliberate self poisoning; repeaters } \\
\text { ( } \geqslant 1 \text { attempt) and first timers; } 71 \% \text { female }\end{array}$ & $\begin{array}{l}\text { Experimental }(\mathrm{n}=200) \text { : crisis orientated, time limited, task centred social } \\
\text { work at home (problem solving intervention). Control }(\mathrm{n}=200) \text { : routine } \\
\text { service- } 54 \% \text { GP referral, } 33 \% \text { psychiatric referral, } 13 \% \text { other referral }\end{array}$ & 400 & $\begin{array}{c}12 \\
\text { months }\end{array}$ & 3 \\
\hline $\begin{array}{l}\text { Hawton et al } \\
\quad(\mathrm{UK}, 1987)^{31}\end{array}$ & $\begin{array}{l}\text { Patients over } 16 \text { years admitted to general hospital } \\
\text { for self poisoning; } 31 \% \text { repeaters; } 66 \% \text { female }\end{array}$ & $\begin{array}{l}\text { Experimental }(n=41) \text { : outpatient problem orientated therapy by } \\
\text { non-medical clinicians. Control }(n=39) \text { : GP care (for example, individual } \\
\text { support, marital therapy) after advice from clinician }\end{array}$ & 80 & $\begin{array}{c}12 \\
\text { months }\end{array}$ & 3 \\
\hline $\begin{array}{l}\text { Salkovskis et al } \\
\quad(\mathrm{UK}, 1990)^{32}\end{array}$ & $\begin{array}{l}\text { Patients aged } 16-65 \text { years (mean } 27.5 \text { ) referred by } \\
\text { duty psychiatrist after antidepressant self } \\
\text { poisoning assessed in A\&E department; all } \\
\text { repeaters with high risk of further repetition; } 50 \% \\
\text { female }\end{array}$ & $\begin{array}{l}\text { Experimental }(n=12) \text { : domicillary cognitive behavioural problem solving } \\
\text { treatment. Control }(n=8) \text { : treatment as usual (GP care) }\end{array}$ & 20 & $\begin{array}{c}12 \\
\text { months }\end{array}$ & 3 \\
\hline $\begin{array}{l}\text { McLeavey et al } \\
\quad \text { (Ireland, 1994) }^{33}\end{array}$ & $\begin{array}{l}\text { Patients aged } 15-45 \text { years (mean 24.4) admitted to } \\
\text { A\&E department after self poisoning; } 35.6 \% \\
\text { repeaters; } 74 \% \text { female }\end{array}$ & $\begin{array}{l}\text { Experimental }(n=19) \text { : interpersonal problem solving skills training. }{ }^{34} \\
\text { Control }(n=20) \text { : brief problem solving therapy }{ }^{35}\end{array}$ & 39 & $\begin{array}{c}12 \\
\text { months }\end{array}$ & 1 \\
\hline \multicolumn{6}{|c|}{ Intensive care plus outreach $v$ standard care } \\
\hline $\begin{array}{l}\text { Chowdhury et al } \\
(\text { UK, 1973) }\end{array}$ & $\begin{array}{l}\text { Patients (all repeaters) admitted to general hospital } \\
\text { after deliberate self harm; } 57 \% \text { female }\end{array}$ & $\begin{array}{l}\text { Experimental }(\mathrm{n}=71) \text { : special aftercare-regular outpatient appointments; } \\
\text { patients also seen without appointments; home visits to patients who } \\
\text { missed appointments; emergency } 24 \text { hour telephone access. Control } \\
(\mathrm{n}=84) \text { : normal aftercare- outpatient appointment with psychiatrist } \\
\text { and/or social worker; non-attenders not pursued }\end{array}$ & 155 & 6 months & 1 \\
\hline Welu (USA, 1977) ${ }^{37}$ & $\begin{array}{l}\text { Suicide attempters over } 16 \text { years brought to } A \& E \\
\text { department; } 60 \% \text { repeaters; \% female not given }\end{array}$ & $\begin{array}{l}\text { Experimental }(n=63) \text { : special outreach programme-community mental } \\
\text { health team contacted patient immediately after discharge; home visit } \\
\text { arranged; weekly/twice weekly contact with therapist. Control }(n=57) \text { : } \\
\text { routine care-appointment for evaluation at the community mental } \\
\text { health centre next day at request of treating physician }\end{array}$ & $120(1)$ & 4 months & 1 \\
\hline $\begin{array}{l}\text { Hawton et al } \\
(\mathrm{UK}, 1981)^{38}\end{array}$ & $\begin{array}{l}\text { Patients } \geqslant 16 \text { years (mean } 25.3 \text { ) admitted to } \\
\text { general hospital after deliberate self poisoning; } \\
32 \% \text { repeaters; } 70 \% \text { female }\end{array}$ & $\begin{array}{l}\text { Experimental }(n=48) \text { : domicillary therapy (brief problem orientated) as } \\
\text { often as therapist thought necessary; open telephone access to general } \\
\text { hospital service. Control }(n=48) \text { : outpatient therapy once a week in } \\
\text { outpatient clinic in general hospital }\end{array}$ & 96 & $\begin{array}{c}12 \\
\text { months }\end{array}$ & 3 \\
\hline $\begin{array}{l}\text { Allard et al } \\
\quad \text { (Canada, 1992) }^{39}\end{array}$ & $\begin{array}{l}\text { Patients seen in A\&E department for suicide } \\
\text { attempt; } 50 \% \text { repeaters; } 55 \% \text { female }\end{array}$ & $\begin{array}{l}\text { Experimental ( } n=76) \text { : intensive intervention-schedule of visits was } \\
\text { arranged including at least one home visit; therapy provided when } \\
\text { needed; reminders (telephone or written) and home visits made if } \\
\text { appointments missed. Control }(n=74) \text { : treatment by another staff team } \\
\text { in the same hospital }\end{array}$ & $150(24)$ & $\begin{array}{c}12 \\
\text { months }\end{array}$ & 3 \\
\hline $\begin{array}{l}\text { Van Heeringen et al } \\
\quad\left(\text { Belgium, 1995) }{ }^{40}\right.\end{array}$ & $\begin{array}{l}\text { Patients } \geqslant 15 \text { years treated in A\&E department } \\
\text { after suicide attempt; } 30 \% \text { repeaters; } 43 \% \text { female }\end{array}$ & $\begin{array}{l}\text { Experimental }(n=258) \text { : special care-home visits by nurse to patients } \\
\text { who did not keep outpatient appointments, reasons for not attending } \\
\text { discussed and patient encouraged to attend. Control }(n=258) \text { : outpatient } \\
\text { appointments only; non-compliant patients not visited }\end{array}$ & $516(125)$ & $\begin{array}{c}12 \\
\text { months }\end{array}$ & 3 \\
\hline $\begin{array}{l}\text { Van der Sande et al } \\
\text { (Netherlands, } \\
\text { 1997) }\end{array}$ & $\begin{array}{l}\text { Patients } \geqslant 16 \text { years (mean } 36.3 \text { ) admitted to } \\
\text { hospital after suicide attempt; } 73 \% \text { repeaters; } \\
66 \% \text { female }\end{array}$ & $\begin{array}{l}\text { Experimental }(n=140) \text { : brief psychiatric unit admission, encouraging } \\
\text { patients to contact unit on discharge; outpatient therapy plus } 24 \text { hour } \\
\text { emergency access to unit. Control ( }(n=134) \text { : usual care-25\% admitted } \\
\text { to hospital, } 65 \% \text { outpatient referral }\end{array}$ & 274 & $\begin{array}{c}12 \\
\text { months }\end{array}$ & 3 \\
\hline $\begin{array}{l}\text { Morgan et al } \\
(\mathrm{UK}, 1993)^{21}\end{array}$ & $\begin{array}{l}\text { Mean age } 30 \text { years; patients admitted after first } \\
\text { episode of deliberate self harm; \% female not } \\
\text { given }\end{array}$ & $\begin{array}{l}\text { Experimental }(n=101) \text { : standard care plus green card (emergency card } \\
\text { indicating that doctor was available and how to contact them). Control } \\
(n=111): \text { standard care-for example, referral back to primary } \\
\text { healthcare team, psychiatric inpatient admission }\end{array}$ & 112 & $\begin{array}{c}12 \\
\text { months }\end{array}$ & 3 \\
\hline $\begin{array}{l}\text { Cotgrove et al } \\
\quad(\mathrm{UK}, 1995)^{22}\end{array}$ & $\begin{array}{l}\text { Patients aged } 12.2-16.7 \text { years (mean } 14.9 \text { ) } \\
\text { admitted after deliberate self harm; \% repeaters } \\
\text { not given; } 85 \% \text { female }\end{array}$ & $\begin{array}{l}\text { Experimental }(n=47) \text { : standard care plus green card (emergency } \\
\text { card) - green card acted as passport to readmission into paediatric ward } \\
\text { in local hospital. Control ( } n=58) \text { : standard follow up treatment from } \\
\text { clinic or child psychiatry department }\end{array}$ & 105 & $\begin{array}{c}12 \\
\text { months }\end{array}$ & 1 \\
\hline \multicolumn{6}{|c|}{ Dialectical behaviour therapy $v$ standard aftercare } \\
\hline $\begin{array}{l}\text { Linehan et al } \\
\quad(\text { USA, 1991) }\end{array}$ & $\begin{array}{l}\text { Patients aged } 18-45 \text { years who had self harmed } \\
\text { within } 8 \text { weeks before entering study; all female; } \\
\text { all multiple repeaters of self harm }\end{array}$ & $\begin{array}{l}\text { Experimental }(n=32) \text { : dialectical behaviour therapy (individual and group } \\
\text { work) for } 1 \text { year; telephone access to therapist. Control }(n=31) \text { : } \\
\text { treatment as usual; } 73 \% \text { individual psychotherapy }\end{array}$ & $63(24)$ & $\begin{array}{c}12 \\
\text { months }\end{array}$ & 3 \\
\hline \multicolumn{6}{|c|}{ Inpatient behaviour therapy $v$ inpatient insight orientated therapy } \\
\hline $\begin{array}{l}\text { Liberman and Eckman } \\
\quad(\text { USA, 1981) }\end{array}$ & $\begin{array}{l}\text { Patients (mean (range) age } 29.7 \text { (18-47) years) all } \\
\text { repeaters; patients referred by psychiatric } \\
\text { emergency service or hospital A\&E department } \\
\text { after deliberate self harm; } 67 \% \text { female }\end{array}$ & $\begin{array}{l}\text { Experimental }(n=12) \text { : inpatient treatment with behaviour therapy. Control } \\
(n=12) \text { : inpatient treatment with insight orientated therapy; both groups } \\
\text { received individual and group therapy plus aftercare at community } \\
\text { mental health centre or with private therapist }\end{array}$ & 24 & $\begin{array}{c}24 \\
\text { months }\end{array}$ & 2 \\
\hline \multicolumn{6}{|c|}{ Same therapist (continuity of care) $v$ different therapist (change of care) } \\
\hline $\begin{array}{l}\text { Torhorst et al } \\
\quad(\text { Germany, 1987) })^{28}\end{array}$ & $\begin{array}{l}\text { Patients referred to toxological department of } \\
\text { Technical University Munich after deliberate self } \\
\text { poisoning; } 48 \% \text { repeaters; } 62 \% \text { female }\end{array}$ & $\begin{array}{l}\text { Experimental }(n=68) \text { : continuity of care-therapy with same therapist } \\
\text { who assessed patient in hospital after attempt. Control }(n=73) \text { : change } \\
\text { of care-therapy with different therapist than seen at hospital } \\
\text { assessment }\end{array}$ & $141(8)$ & $\begin{array}{c}12 \\
\text { months }\end{array}$ & 2 \\
\hline \multicolumn{6}{|c|}{ General hospital admission v discharge } \\
\hline $\begin{array}{l}\text { Waterhouse and Platt } \\
(\text { UK, 1990) })^{43}\end{array}$ & $\begin{array}{l}\text { Patients } \geqslant 16 \text { years (mean } 30.3 \text { ) admitted to A\&E } \\
\text { department for deliberate self harm; } 36 \% \\
\text { repeaters; } 63 \% \text { female }\end{array}$ & $\begin{array}{l}\text { Experimental }(n=38) \text { : general hospital admission. Control }(n=39) \text { : } \\
\text { discharge from hospital; on discharge both groups advised to contact } \\
\text { GP if they needed further help }\end{array}$ & 77 & 16 weeks & 3 \\
\hline \multicolumn{6}{|l|}{ Flupenthixol $v$ placebo } \\
\hline $\begin{array}{l}\text { Montgomery et al (UK, } \\
1979)^{26}\end{array}$ & $\begin{array}{l}\text { Patients aged } 18-68 \text { years (mean } 35.3 \text { ) admitted } \\
\text { after suicidal act; all repeaters; } 70 \% \text { female }\end{array}$ & $\begin{array}{l}\text { Experimental }(n=18): 20 \mathrm{mg} \text { intramuscular flupenthixol deconate for } \\
6 \text { months. Control }(n=19) \text { : placebo for } 6 \text { months }\end{array}$ & $37(7)$ & 6 months & 3 \\
\hline \multicolumn{6}{|l|}{ Antidepressants $v$ placebo } \\
\hline $\begin{array}{l}\text { Hirsch et al (UK, } \\
1982)^{16} \mathrm{R} \text { Draper, } \\
\text { S Hirsch (personal } \\
\text { communication) }\end{array}$ & $\begin{array}{l}\text { Patients aged } 16-65 \text { years admitted after deliberate } \\
\text { self poisoning; \% repeaters and \% female not } \\
\text { given }\end{array}$ & $\begin{array}{l}\text { Experimental }(n=76) \text { : antidepressants-either } 30-60 \mathrm{mg} \text { mianserin for } \\
6 \text { weeks or } 75-150 \mathrm{mg} \text { nomifensine for } 6 \text { weeks. Control }(n=38) \text { : } \\
\text { placebo for } 6 \text { weeks }\end{array}$ & 114 & 12 weeks & 3 \\
\hline $\begin{array}{c}\begin{array}{l}\text { Montgomery et al } \\
(\mathrm{UK}, 1983)^{44}\end{array}\end{array}$ & $\begin{array}{l}\text { Patients with personality disorders (mean age } 35.7 \\
\text { years) admitted to medical ward after deliberate } \\
\text { self harm; all repeaters; } 66 \% \text { female }\end{array}$ & $\begin{array}{l}\text { Experimental }(n=17) \text { : mianserin } 30 \mathrm{mg} \text { for } 6 \text { months. Control }(\mathrm{n}=21) \text { : } \\
\text { placebo }\end{array}$ & 38 & 6 months & 3 \\
\hline \multicolumn{6}{|c|}{ Long term therapy $v$ short term therapy } \\
\hline $\begin{array}{l}\text { Torhorst et al } \\
{\text { (Germany, } 1988)^{45}}^{4}\end{array}$ & $\begin{array}{l}\text { All patients repeaters who had deliberately self } \\
\text { poisoned; \% female not given }\end{array}$ & $\begin{array}{l}\text { Experimental }(n=40) \text { : Iong term therapy-one therapy session a month } \\
\text { for } 12 \text { months. Control }(n=40) \text { : short term therapy }-12 \text { weekly therapy } \\
\text { sessions for } 3 \text { months; all participants had brief crisis intervention } \\
\text { ( } 3 \text { days) in hospital }\end{array}$ & 80 & $\begin{array}{c}12 \\
\text { months }\end{array}$ & 2 \\
\hline
\end{tabular}

*1=poorest quality; $3=$ best quality. 
Table 2 Summary of outcome data on repetition of deliberate self harm

\begin{tabular}{|c|c|c|}
\hline \multirow[b]{2}{*}{ Category, trial } & \multicolumn{2}{|c|}{$\begin{array}{c}\text { Proportion (\%) of participants who repeated behaviour } \\
\text { during follow up }\end{array}$} \\
\hline & Experimental & Control \\
\hline \multicolumn{3}{|l|}{ Problem solving therapy $\boldsymbol{v}$ standard aftercare } \\
\hline Gibbons et al $(1978)^{30}$ & $27 / 200(13.5)$ & 29/200 (14.5) \\
\hline Hawton et al $(1987)^{31}$ & $3 / 41(7.3)\left(1^{\star}\right)$ & $6 / 39(15.4)\left(0^{\star}\right)$ \\
\hline Salkovskis et al $(1990)^{32}$ & $3 / 12(25.0)$ & $4 / 8(50.0)$ \\
\hline McLeavey et al $(1994)^{33}$ & $2 / 19(10.5)$ & $5 / 20(25.0)$ \\
\hline \multicolumn{3}{|l|}{ Intensive care plus outreach $v$ standard care } \\
\hline Chowdhury et al $(1973)^{36}$ & $17 / 71(23.9)$ & 19/84 (22.6) \\
\hline Welu $(1977)^{37}$ & $3 / 62(4.8)$ & 9/57 (15.8) \\
\hline Hawton et al $(1981)^{38}$ & $5 / 48(10.4)\left(0^{*}\right)$ & $7 / 48(14.6)\left(0^{\star}\right)$ \\
\hline Allard et al $(1992)^{39}$ & $22 / 63(34.9)\left(3^{*}\right)$ & $19 / 63(30.2)\left(1^{\star}\right)$ \\
\hline Van Heeringen et al $(1995)^{40}$ & $21 / 196(10.7)\left(6^{\star}\right)$ & $34 / 195(17.4)\left(7^{*}\right)$ \\
\hline Van der Sande et al $(1997)^{41}$ & $24 / 140(17.1)$ & 20/134 (14.9) \\
\hline \multicolumn{3}{|l|}{ Emergency card $v$ standard aftercare } \\
\hline Morgan et al $(1993)^{21}$ & $5 / 101(5.0)\left(0^{\star}\right)$ & $12 / 111(10.8)\left(0^{*}\right)$ \\
\hline Cotgrove et al $(1995)^{22}$ & $3 / 47(6.4)$ & $7 / 58(12.1)$ \\
\hline \multicolumn{3}{|c|}{ Dialectical behaviour therapy $v$ standard aftercare } \\
\hline Linehan et al $(1991)^{27} \dagger$ & $5 / 19(26.3)$ & $12 / 20(60.0)$ \\
\hline \multicolumn{3}{|c|}{ Inpatient behaviour therapy $v$ inpatient insight orientated therapy } \\
\hline Liberman and Eckman (1981) ${ }^{42}$ & $2 / 12(16.7)$ & $3 / 12(25.0)$ \\
\hline \multicolumn{3}{|c|}{ Same therapist (continuity of care) $v$ different therapist (change of care) } \\
\hline Torhorst et al $(1987)^{28}$ & 12/68 (17.6) & $4 / 73(5.5)$ \\
\hline \multicolumn{3}{|l|}{ General hospital admission $v$ discharge } \\
\hline Waterhouse and Platt (1990) ${ }^{43}$ & $3 / 38(7.9)$ & $4 / 39(10.3)$ \\
\hline \multicolumn{3}{|l|}{ Flupenthixol v placebo } \\
\hline Montgomery et al $(1979)^{26}$ & $3 / 14(21.4)$ & $12 / 16(75.0)$ \\
\hline \multicolumn{3}{|l|}{ Antidepressants v placebo } \\
\hline $\begin{array}{l}\text { Hirsch et al (1982), }{ }^{16} \mathrm{R} \text { Draper, S Hirsch } \\
\text { (personal communication) }\end{array}$ & $16 / 76(21.1)\left(0^{\star}\right)$ & $5 / 38(13.2)\left(0^{*}\right)$ \\
\hline Montgomery et al $(1983)^{44}$ & $8 / 17(47.1)$ & 12/21 (57.1) \\
\hline \multicolumn{3}{|l|}{ Long term therapy $v$ short term therapy } \\
\hline Torhorst et al $(1988)^{45}$ & $9 / 40(22.5)$ & $9 / 40(22.5)$ \\
\hline
\end{tabular}

was unclear in 12 trials, was indicated but no further details given in seven trials, and was fully reported in one trial.

A total of 2641 patients were randomised in the 20 trials, and outcome data regarding repetition of deliberate self harm during follow up were available for 2452. The results of the individual studies in terms of repetition of deliberate self harm during follow up are shown in table 2. Also shown is the number of suicides, when these were reported.

\section{Summary odds ratios}

The summary odds ratios for each of the treatment categories that included more than one study are shown in the figure, which also indicates the total number of patients within each category.

Problem solving therapy versus standard aftercare-All four studies reported reduced repetition of deliberate self harm in patients in the experimental groups. The summary odds ratio of 0.73 (95\% confidence interval 0.45 to 1.18 ), however, was not significant. It should be noted that the effect size was smallest in the largest trial $^{30}$ in this category. The trial which involved two forms of problem solving was included here because the control treatment of brief problem solving was standard treatment by the time this study was conducted..$^{33}$ Omission of this trial, which was also the only one in this category not given the highest rating of quality of concealment of allocation, made little difference to the summary odds ratio $(0.78 ; 0.47$ to 1.29$)$.
Intensive intervention plus outreach versus standard aftercare-There was no consistent direction of effect among studies in this group (see figure). Inclusion of only those trials with the highest quality of concealment of allocation did not greatly alter the summary odds ratio $(0.86 ; 0.60$ to 1.23$)$.

Emergency card versus standard aftercare-In both studies in this comparison there was a tendency towards less repetition of self harm in the experimental group, but the summary odds ratio 0.45 (0.19 to 1.07) was not significant. The odds ratio was similar when only the results of the trial with the highest quality rating were analysed $(0.43 ; 0.15$ to 1.27$)$.

Dialectical behaviour therapy versus standard aftercare - In this study ${ }^{27}$ there was a significantly lower rate of repetition of self harm during follow up in patients who received dialectical behaviour therapy (see table $2 ; 0.24 ; 0.06$ to 0.93 ; number needed to treat $=3$ ). This comparison, however, was restricted to a subgroup of randomly assigned patients which was smaller than that which entered the original trial. ${ }^{46}$

Inpatient behaviour therapy versus inpatient insight orientated therapy-The small sample size of the single study in this comparison (see table 2) precluded meaningful conclusions from the odds ratio analysis $(0.60$; 0.08 to 4.45$)$.

Same therapist versus different therapist-The repetition rate in the group of patients who received aftercare from the same person who assessed them in hospital after their initial episode of deliberate self harm was significantly higher than that of patients who had a change of clinician (see table $2 ; 3.70 ; 1.13$ to 12.09 ). The authors reported, however, that despite randomisation there were several imbalances between the experimental and control groups, resulting in a greater prevalence of risk factors for repetition in the experimental group. It is of note that continuity of therapist resulted in 48/68 (71\%) patients attending at least one outpatient treatment session compared with $34 / 73(47 \%)$ patients in the control group $(2.75 ; 1.37$ to 5.52$)$.

General hospital admission versus discharge-The odds ratio from the one study in this category (see table 2) did not indicate a beneficial effect of general hospital admission after deliberate self harm $(0.75 ; 0.16$ to $3.60)$. Only $15 \%$ of patients referred, however, were eligible for inclusion in the study as only those attempters at low risk and without immediate medical or psychiatric needs could be considered for discharge without treatment. The follow up period was relatively short.

Flupenthixol versus placebo-There was a significant reduction in repetition of deliberate self harm in patients receiving flupenthixol (see table 2; 0.09; 0.02 to 0.50 ; number needed to treat $=2$ ). The trial was relatively small and all the patients were repeaters.

Antidepressants versus placebo-The summary odds ratio for the two studies in this category (see figure) indicated no apparent benefit regarding repetition of deliberate self harm for patients treated with mianserin or nomifensine compared with placebo (1.19; 0.53 to 2.67).

Long term therapy versus short term therapy-There was no indication that long term therapy was more effective in terms of preventing repetition than short term therapy for patients with a history of self harm (see table $2 ; 1.0 ; 0.35$ to 2.86 ). 


\section{Discussion}

The results of this systematic review indicate that currently there is insufficient evidence on which to make firm recommendations about the most effective forms of treatment for patients who have recently deliberately harmed themselves. This is a serious situation given the size of the problem of deliberate self harm throughout the world ${ }^{10-13}$ and the importance of dealing with the problem to prevent suicide. ${ }^{3}$

In nearly all trials the subjects were recruited after attendance at a general hospital because of deliberate self harm. Some trials included only patients who had poisoned themselves, who constitute most of the patients who deliberately harm themselves ${ }^{10}$; others included patients who had poisoned themselves and those who had injured themselves, whereas some did not specify the method of self harm. Most of the studies focused on patients who could be treated as outpatients. Patients who, for example, required psychiatric hospital inpatient care because of severe mental illness or serious risk of suicide, or both, were excluded, but these comprise the minority of patients who harm themselves and who present to general hospitals. ${ }^{10}$ The studies examined are therefore of relevance to a large proportion of patients who deliberately harm themselves who will be treated in the community. Most patients in the studies had a history of episodes of self harm, and in seven trials the whole sample consisted of such patients. ${ }^{26} 2732364244$ Only one study included only patients with no history of self harm. ${ }^{21}$ In view of the considerable problem of deliberate self harm in adolescents in many countries ${ }^{117}$ it is surprising that only one trial focused on this specific clinical population. ${ }^{22}$

\section{Shortcomings of trials}

The comparison intervention for most of the studies of psychosocial intervention was standard care. In some studies details of this care were not provided, particularly in terms of treatment content. Future studies in which standard care is included should define precisely the nature of the treatment patients received. The dependent variable studied in this review-namely, repetition of self harm-was not consistently defined and measured in a standard way across all studies. In most studies repetition was based on hospital referral for further deliberate self harm, whereas in some studies interviews with patients and other informants also identified episodes of self harm which did not result in hospital referral.

The main problem with nearly all trials in this study is that they included far too few subjects to have the statistical power to detect clinically meaningful differences in rates of repetition of deliberate self harm between experimental and control treatments, if such differences existed. The number needed is a function of both the expected rate of repetition (that is, that in the control group) and the size of the difference. If the predicted rate were $10 \%$ in the experimental group versus $15 \%$ in the control, with $\alpha$ set at 0.05 and $\beta$ set at $0.2,1560$ subjects would be required in each treatment group, whereas if the rates were 20\% and 30\%, 293 subjects would be required in each group. ${ }^{48}$ Even when the results from similar trials were synthesised with meta-analytical techniques there were insufficient numbers of patients to detect such differences. The

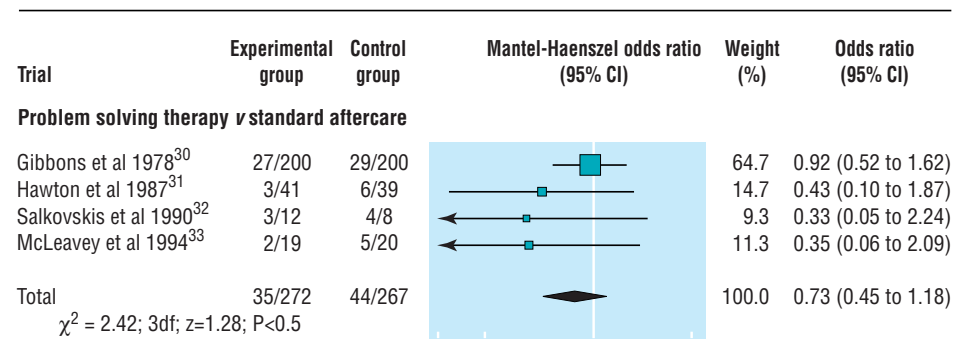

Intensive care plus outreach v standard aftercare

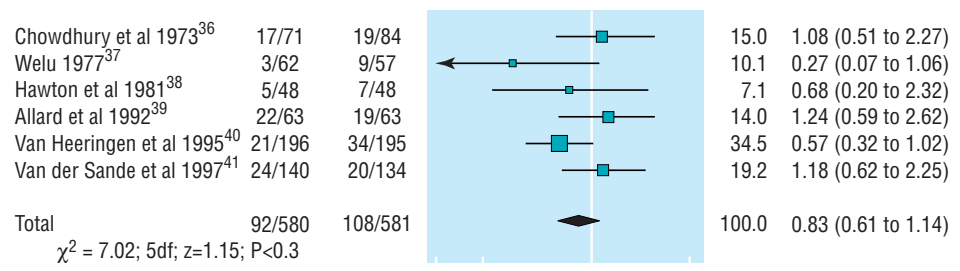

Emergency card $v$ standard aftercare
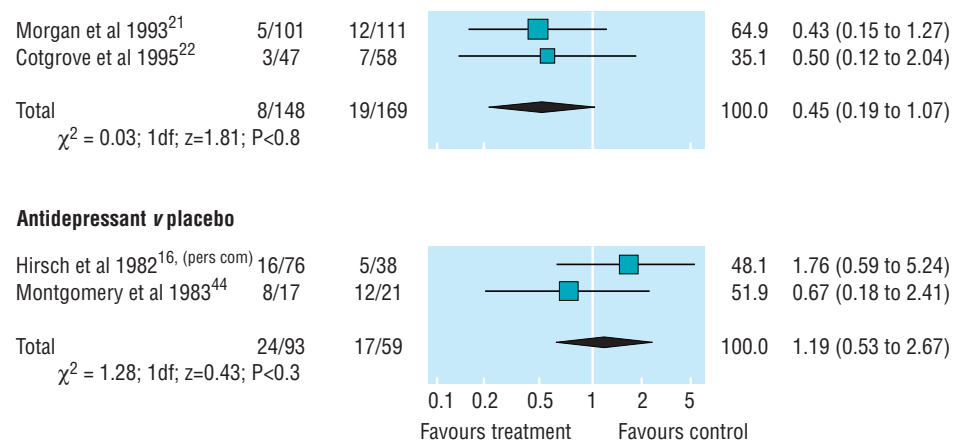

Summary odds ratios for repetition of deliberate self harm during follow up in treatment categories for which there was more than one trial

only significant findings have come from smaller studies, which may reflect publication bias. ${ }^{49}$

\section{Further research indicated}

Promising results were found for problem solving therapy, which is a brief and reasonably easily taught form of treatment. ${ }^{50}$ Although a clinically insignificant difference was found in the biggest trial, ${ }^{30}$ a larger trial of this treatment is indicated. There were also trends favouring provision of an emergency access card in addition to standard aftercare but again a larger trial is required, including specific attention to what part the card might play as only a small minority of patients actually used the facility provided by possession of the card. $^{21} 22$

A larger replicative trial is required of the promising single study of dialectical behaviour therapy (which is similar to cognitive behaviour therapy) in women with borderline personality disorder who have a history of multiple episodes of deliberate self harm. ${ }^{27}$ As the intervention is intensive, ${ }^{51}$ development and evaluation of a shorter form of this treatment more suited to provision of general psychiatric services and investigation of its efficacy in men are needed.

The positive result of depot neuroleptic medication in a single small study of patients who repeatedly harm themselves ${ }^{26}$ suggests that this treatment should be subjected to further evaluation in a larger study, 
Key messages

- A systematic review of the effectiveness of psychosocial and drug treatments of patients who deliberately harm themselves identified 20 randomised controlled trials in which repetition of self harm was reported as an outcome

- Promising results were found for problem solving therapy, provision of a card to allow patients to make emergency contact with services, depot flupenthixol for recurrent self harm, and long term psychological therapy for female patients with borderline personality disorder and recurrent self harm

- Assertive outreach can help to keep patients in treatment

- Nearly all the trials included too few patients to detect clinically significant differences in repetition of self harm, and even synthesis of results by meta-analysis did not have the power to detect such differences

- There is an urgent need for large trials of promising therapies for this substantial clinical population

although reluctance of patients to accept depot medication, side effects, and other practical and ethical implications, may limit its applicability. There was little indication that intensive intervention plus outreach was effective. In one relatively large study in this group, which evaluated community follow up of patients who did not attend outpatient appointments, ${ }^{40}$ there was a significant increase in outpatient attendance from $42.5 \%$ before the home visit $(39.8 \%$ in the control group) to $51.2 \%$ after the visit (odds ratio $1.58 ; 1.15$ to 2.33) and a near significant difference in repetition of deliberate self harm of $10.7 \%$ compared with $17.4 \%$ $(0.57 ; 0.32$ to 1.02$)$. Home treatment was also found substantially to increase the rate of take up of treatment in another trial in this review. ${ }^{38}$ Assertive outreach for poorly compliant patients may, therefore, be a necessary component in maximising the delivery of any treatment that is shown to be effective.

There was no evidence that antidepressants were generally effective in preventing repetition of behaviour in patients who deliberately harm themselves. One of the drugs investigated, however, is no longer available (nomifensine) and the other (mianserin) is now little used. This review does not give any indication of whether other antidepressants could be of benefit.

Repetition of deliberate self harm has been the sole outcome variable investigated in this review. It will be important to determine whether there is evidence of benefits with regard to other outcomes (for example, depression or problem resolution), although the data for these factors, when reported, were often inadequate for meta-analysis. The groups of patients were often heterogeneous in term of sex, age, and presenting problems. Further work in this specialty should examine the efficacy of interventions according to such factors.

\section{Conclusion}

At present, evidence is lacking to indicate the most effective forms of treatment for patients who deliberately harm themselves. This is a serious situation given the size of the population at risk and the risks of subsequent self harm, including suicide. Large trials are required of the interventions shown in small trials to be of possible benefit. There is also a need for development of further treatment approaches informed by current knowledge about the psychosocial and biological characteristics of these patients and the socioeconomic and sociocultural context of the behaviour.

This study was carried out under the auspices of the Depression, Anxiety and Neurosis Review Group of the Cochrane Collaboration. Updated versions of the review will appear in the Cochrane Controlled Trials Register in the future. We thank Rachel Churchill and Drs Douglas Altman, Clive Adams, John Geddes, and Henry McQuay for advice. We also thank the several authors who supplied us with unpublished information regarding their trials.

Contributors: $\mathrm{KH}$ initiated the study and together with EA and ET designed the original protocol, coordinated the study, and analysed the data. EA and ET conducted the electronic searching and the data abstraction. All the authors (KH, EA, ET, SB, EF, RG, DG, PH, KvH, AH, DO, IS, LT-B) contributed to the revision of the protocol, hand searching of journals, and the writing of the paper, which was initially drafted by KH and ET. $\mathrm{EF}, \mathrm{PH}, \mathrm{AH}, \mathrm{KvH}$, and IS carried out the quality assessments. $\mathrm{KH}$ is guarantor for the paper.

Funding: Anglia and Oxford Research and Development Committee.

Conflict of interest: None.

World Health Organisation. Health-for-all targets. The health policy for Europe. Summary of the updated edition. Copenhagen: World Health Organisation, 1992. (EUR ICP HSC 013.)

2 Department of Health. The health of the nation: a strategy for health in England. London: HMSO, 1992. (Cmnd 1986.)

3 Gunnell D, Frankel S. Prevention of suicide: aspirations and evidence. BMJ 1994;308:1227-33

4 Hawton K, Fagg J. Suicide, and other causes of death, following attempted suicide. Br J Psychiatry 1988;152:751-61.

5 Suokas J, Lönnqvist J. Outcome of attempted suicide and psychiatric consultation: risk factors and suicide during a five-year follow-up. Acta Psychiatr Scand 1991;84:545-9.

6 Nordentoft M, Breum L, Munck LR, Nordestgaard AG, Hunding A, Laursen Bjaeldager PA. High mortality by natural and unnatural causes: a 10 year follow up study of patients admitted to a poisoning treatment centre after suicide attempts. BMJ 1993;306:1637-41.

7 Ovenstone IMK, Kreitman N. Two syndromes of suicide. Br J Psychiatry 1974;124:336-45.

8 Foster T, Gillespie K, McClelland R. Mental disorders and suicide in Northern Ireland. Br J Psychiatry 1997;170:447-52

9 Bancroft J, Marsack P. The repetitiveness of self-poisoning and self-injury Br J Psychiatry 1977;131:394-9.

10 Hawton K, Fagg J, Simkin S, Bale E, Bond A. Trends in deliberate self-harm in Oxford, 1985-1995: implications for clinical services and the prevention of suicide. J Psychiatry 1997;171:556-60.

11 Schmidtke A, Bille-Brahe U, Deleo D, Kerkhof A, Bjerke T, Crepet P, et al. Attempted suicide in Europe: rates, trends, and sociodemographic characteristics of suicide attempters during the period 1989-1992. Results of the WHO/EURO multicentre study on parasuicide. Acta Psychiatr Scand 1996;93:327-38.

12 Wexler L, Weissman, MM, Kasl, SV. Suicide attempts 1970-75: updating a United States study and comparisons with international trends. Br J Psychiatry 1978;132:180-5.

13 Davis, AT, Kosky RJ. Attempted suicide in Adelaide and Perth: changing rates for males and females, 1971-1987. Med J Aust 1991;154:666-70.

14 Bialas MC, Reid PG, Beck JH, Lazarus JH, Smith PM, Scorer RC, et al. Changing patterns of self-poisoning in a UK health district. QJ Med 1996;89:893-901.

15 McLoone P, Crombie IK. Hospitalization for deliberate self-poisoning in Scotland from 1981 to 1993: trends in rates and types of drugs used. $\mathrm{BrJ}$ Psychiatry 1996;169:81-6.

16 Hirsch SR, Walsh C, Draper R. Parasuicide: a review of treatment interventions. J Affective Disord 1982;4:299-311.

17 Dew MA, Bromet JE, Brent D, Greenhouse JB. A quantitative literature review of the effectiveness of suicide prevention centers. J Consult Clin Psychol 1987;55:229-44.

18 Hawton K. Attempted suicide. In: Clarke DM, Fairburn CG, eds. Science and practice of cognitive behaviour therapy. Oxford: Oxford University Press, 1997:285-312

19 Van der Sande R, Buskens E, Allart E, van der Graaf Y, van Engeland H. Psychosocial intervention following suicide attempt: a systematic review of treatment interventions. Acta Psychiatr Scand 1997;96:43-50.

20 Cochrane Collaboration. The Cochrane Controlled Trials Register. In The Cochrane Library, Issue 4. Oxford: Update Software, 1997.

21 Morgan HG, Jones EM, Owen JH. Secondary prevention of non-fatal deliberate self-harm. The green card study. $\mathrm{Br} \quad J$ Psychiatry 1993;163:111-2.

22 Cotgrove AJ, Zirinsky L, Black D, Weston D. Secondary prevention of attempted suicide in adolescence. J Adolescence 1995;18:569-77.

23 Sackett DL, ed. The Cochrane Collaboration handbook. Oxford: The Cochrane Collaboration, March 1997.

24 Schulz KF, Chalmers I, Hayes RJ, Altman DG. Empirical evidence of bias: dimensions of methodological quality associated with estimates of treatment effects in controlled trials. JAMA 1995;273:408-12.

25 Update Software. ReuMan 3.0. Oxford: Update Software, 1996 
26 Montgomery SA, Montgomery DB, Jayanthi-Rani S, Roy DH, Shaw PJ, McAuley R. Maintenance therapy in repeat suicidal behaviour: a placebo controlled trial. Proceedings of 10th International Congress for Suicide Prevention and Crisis Intervention; 1979; Ottawa: 227-9.

27 Linehan MM, Armstrong HE, Suarez A, Allmari D, Heard HL. Cognitivebehavioral treatment of chronically parasuicidal borderline patients. Arch Gen Psychiatry 1991:48:1060-4.

28 Torhorst A, Möller HJ, Bürk F, Kurz A, Wächtler C, Lauter H. The psychiatric management of parasuicide patients: a controlled clinical stud comparing different strategies of outpatient treatment. Crisis 1987;8:53-61.

29 Patsiokas AT, Clum GA. Effects of psychotherapeutic strategies in the treatment of suicide attempters. Psychotherapy 1985;22:281-90.

30 Gibbons JS, Butler J, Urwin P, Gibbons JL. Evaluation of a social work service for self-poisoning patients. Br J Psychiatry 1978;133:111-8.

31 Hawton K, McKeown S, Day A, Martin P, O'Connor M, Yule J. Evaluation of out-patient counselling compared with general practitioner care following overdoses. Psychol Med 1987;17:751-61.

32 Salkovskis PM, Atha C, Storer D. Cognitive-behavioural problem solving in the treatment of patients who repeatedly attempt suicide: a controlled trial. Br J Psychiatry 1990;157:871-6.

33 McLeavey BC, Daly RJ, Ludgate JW, Murray CM. Interpersonal problemsolving skills training in the treatment of self-poisoning patients. Suicide Life Threatening Behav 1994;24:382-94.

34 D'Zurilla TJ, Godfried MR. Problem solving and behaviour modification. JAbnorm Psychol 1971;78:107-26.

35 Hawton K, Catalan J. Attempted suicide: a practical guide to its nature and management. Oxford: Oxford University Press, 1982.

36 Chowdhury N, Hicks RC, Kreitman N. Evaluation of an after-care service for parasuicide (attempted suicide) patients. Soc Psychiatry 1973;8:67-81.

37 Welu TC. A follow-up program for suicide attempters: evaluation of effectiveness. Suicide Life Threatening Behav 1977;7:17-30.

38 Hawton K, Bancroft J, Catalan J, Kingston B, Stedeford A, Welch N Domiciliary and out-patient treatment of self-poisoning patients by medical and non-medical staff. Psychol Med 1981;11:169-77.

39 Allard R, Marshall M, Plante MC. Intensive follow-up does not decrease the risk of repeat suicide attempts. Suicide Life Threatening Behav 1992;22:303-14.
40 Van Heeringen CV, Jannes S, Buylaert H, Hendrick H, de Bacquer D, van Remoortel J. The management of non-compliance with referral to out-patient after-care among attempted suicide patients: a controlled intervention study. Psychol Med 1995;25:963-70.

41 Van der Sande R, van Rooijen E, Buskens E, Allart E, Hawton K, van der Graaf Y, et al. Intensive in-patient and community intervention versus routine care after attempted suicide: a randomised controlled intervention. Br J Psychiatry 1997;171:35-41

42 Liberman RP, Eckman T. Behavior therapy vs. insight-oriented therapy for repeated suicide attempters. Arch Gen Psychiatry 1981;38:1126-30.

43 Waterhouse J, Platt S. General hospital admission in the management of parasuicide: a randomised controlled trial. Br J Psychiatry 1990;156: 236-42.

44 Montgomery SA, Roy D, Montgomery DB. The prevention of recurrent suicidal acts. Br J Clin Pharmacol 1983;15:183-8S.

45 Torhorst A, Möller HJ, Kurz A, Schmid-Bode KW, Lauter H. Comparing a 3-month and a 12-month-outpatient aftercare program for parasuicide repeaters. In: Möller HJ, Schmidtke A, Welz R, eds. Current issues of suicidology. Berlin: Springer-Verlag, 1988:419-24.

46 Linehan MM, Heard HL, Armstrong HE. Naturalistic follow-up of a behavioral treatment for chronically parasuicidal borderline patients. Arch Gen Psychiatry 1993;50:971-4.

47 Hawton K, Fagg J. Deliberate self-poisoning and self-injury in adolescents: a study of characteristics and trends in Oxford, 1976-89. $\mathrm{Br} J$ Psychiatry 1992;161:816-23.

48 Pocock SJ. Clinical trials: a practical approach. Chichester: Wiley, 1983.

49 Light RJ, Pillemer DB. Summing up: the science of reviewing research. Cambridge, Massachusetts: Harvard University Press, 1984.

50 Hawton K, Kirk J. Problem-solving. In: Hawton K, Salkovskis P, Kirk J Clark DM, eds. Cognitive behaviour therapy for psychiatric problems: a practical guide. Oxford: Oxford University Press, 1989:406-26.

51 Linehan MM. Cognitive behavioral treatment of borderline personality disorder. New York: Guilford, 1993.

(Accepted 27 April 1998)

\title{
An epidemiological needs assessment of carotid endarterectomy in an English health region. Is the need being met?
}

\author{
G Ferris, P Roderick, A Smithies, S George, J Gabbay, N Couper, A Chant
}

\begin{abstract}
Objective: To compare the level of provision of carotid endarterectomy (an intervention of proved efficacy for prevention of stroke in patients with symptomatic high grade carotid artery stenosis) with estimates of need.

Design: Comparison of regional, district, and age-sex specific operation rates derived from hospital episode statistics with estimates of need based on demographic and epidemiological data; interviews with regional vascular surgeons and a joint provider-purchaser workshop to discuss implications. Setting: Former Wessex Regional Health Authority, 1991-2 to 1995-6.

Subjects: All residents covered by Wessex region treated for carotid artery reconstruction.

Main outcome measures: Regional, district, and age-sex operation rates as three year average 1993-6 (use) compared with respective estimates of need for carotid endarterectomy among those who presented with symptomatic carotid disease-transient ischaemic attack or minor stroke.

Results: The operation rate more than doubled between 1991-2 and 1995-6, from 35 to 89 per million population, compared with an estimated level of need in the region's general population of 153 per million population (transient ischaemic attack 77 , minor stroke 76 ). The ratio of use to need was 0.47
\end{abstract}

(95\% confidence interval 0.4 to 0.54 ); district ratios were $0.28(0.19-0.38)$ to 0.81 (0.62 to 1.06$)$. The annual use:need ratio rose over the three years 1993-6 from 0.38 to 0.59 . Use:need ratios were lower in elderly and female patients. Providers were keen to develop guidelines for referral and to increase access to diagnostic facilities; purchasers were more reluctant, given the limited impact of this intervention on the incidence of stroke and the relatively high cost of the operation.

Conclusion: Although treatment rates increased in Wessex there is still unmet need. Further research is needed to determine the referral pathways of patients with symptomatic carotid disease for diagnosis and operation and to evaluate strategies to improve access to diagnostic facilities.

\section{Introduction}

Stroke is the third commonest cause of death in the United Kingdom and the commonest cause of physical disability. ${ }^{1}$ Each year 64000 deaths (12\% of all deaths) are attributed to stroke. ${ }^{1}$ Treating and managing patients who have had a stroke is estimated to consume about $4.0 \%$ of NHS resources. ${ }^{2}$

Carotid endarterectomy (the removal of atherosclerotic lesions from the inner wall of the common carotid artery extending up to the internal carotid artery) has a small but significant part to play in
Wessex Institute for Health Research and Development, University of Southampton, Southampton General Hospital, Southampton SO16 6YD

G Ferris, research fellow P Roderick, senior lecturer A Smithies, research fellow S George, senior lecturer J Gabbay, professor

N Couper, research fellow Department of Vascular Surgery, Southampton University Hospitals' Trust, Southampton SO16 6YD

A Chant,

consultant vascular surgeon

continued over

BMJ 1998;317:447-51 